\title{
Portal vein embolisation
}

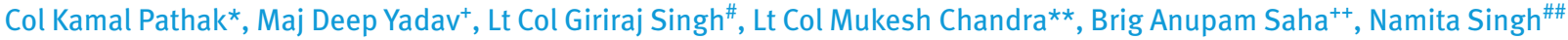

MJAFI 2011;67:274-276

\section{INTRODUCTION}

Hepatic lobar or extended lobar surgeries are now being offered as treatment options in a wide spectrum of focal liver diseases. In recent years, major advances in hepatobiliary surgical techniques have led to improved perioperative outcomes in patients who undergo hepatic resection. A major limitation to satisfactory outcome of such surgeries is relatively low volume of the residual liver. Portal vein embolisation is one of the available options to pre-operatively increase the future liver remnants (FLR). We present one such case where portal vein embolisation was used pre-operatively to increase the volume of FLR prior to liver surgery.

\section{CASE REPORT}

A 45-year-old diabetic serving officer reported to a service hospital with complaints of colicky pain and increasing jaundice for two months. Imaging (USG and MRCP) showed distended gall bladder with oedematous wall with calculus impacted at the neck of gall bladder, mild central intrahepatic biliary radicles and obstruction in the region of hepatic ductal confluence. He was presumed to be a case of Mirizzi's syndrome and taken up for an open cholecystectomy. During surgery the Calot's triangle was seen to be frozen with no mass felt at the porta. The histopathology of the excised gall bladder was chronic cholecystitis. However, the patient remained symptomatic post surgery with the bilirubin showing a steady upward trend. Repeat MRCP was suggestive of stricture at confluence of bile ducts. A triple phase CT scan suggested the presence of an ill defined mass at bile duct confluence with involvement of the right hepatic artery. A diagnosis of hilar cholangiocarcinoma was made and the patient was planned for a right hepatectomy and referred to a tertiary care service hospital.

Calculation of the liver volumes revealed a fatty liver of $1850 \mathrm{cc}$ with the left lobe volume being $518 \mathrm{cc}$ (28\%) (Figure 1).

\footnotetext{
*Senior Advisor (Radiology and Interventional radiology), ${ }^{+}$Graded Specialist (Radiology), MH CTC, Pune, "Classified Specialist (Radiology), ${ }^{\star \star}$ Graded Specialist (Radiology), ${ }^{++}$Consultant, (Surgery and GI Surgery), $A H(R \& R)$, Delhi Cantt., "\# Scientist 'F', Institute of Nuclear Medicine and Allied Sciences, Lucknow Road, Timarpur, New Delhi.
}

Correspondence: Col Kamal Pathak, Senior Advisor (Radiology and Interventional radiology), $\mathrm{MH}$ CTC, Pune - 40.

E-mail:pathakamal@gmail.com

Received: 30.03.2010; Accepted: 07.10.2010

doi: 10.1016/S0377-1237(11)60060-9
The residual liver volume of $518 \mathrm{cc}$ was considered inadequate for surgery. It was decided to perform portal vein embolisation of the right lobe to induce selective hypertrophy of the left lobe to increase the future liver remnants volume.

Patient had conjugated hyperbilirubinemia of $22 \% \mathrm{mg}$. Hence a percutaneous transhepatic biliary drainage (PTBD) was performed and over a period of four weeks, the serum bilirubin dropped to $5.45 \% \mathrm{mg}$. Following the drop in bilirubin level, the patient was taken up for trans-hepatic portal vein embolisation.

The procedure was done under total intravenous anaesthesia. The right portal vein was accessed with a $21 \mathrm{G}$ Chiba needle. A 0.018 " guide wire was placed in the superior mesenteric vein. A 6 Fr sheath was placed in the right portal vein and a portogrom obtained (Figure 2). A 5 Fr Sims catheter was used to enter the branches of the right portal vein and embolisation done with four vials of $700-1000$ and eight vials of $500-700 \mu$ polyvinyl alcohol particles. The posterior division was later coiled with a $7 \mathrm{~mm}$ coil and the right lateral branch with a $6 \mathrm{~mm}$ coil. The track was embolised with a $3 \mathrm{~mm}$ coil. Post-procedure venogram shows filling of the branches of the left portal vein with occlusion of the branches of the right portal vein (Figure 3).

The patient was followed up on Doppler ultrasound. A repeat CECT scan was performed five weeks after the portal vein embolisation. The volume calculations revealed that the left lobe volume had increased to $40 \%$ (Figure 4 ). The patient then underwent a right hepatectomy with excision of caudate lobe and the extrahepatic biliary tree. Postoperatively the patient had cholestasis for six weeks which was presumed to be due to "small for

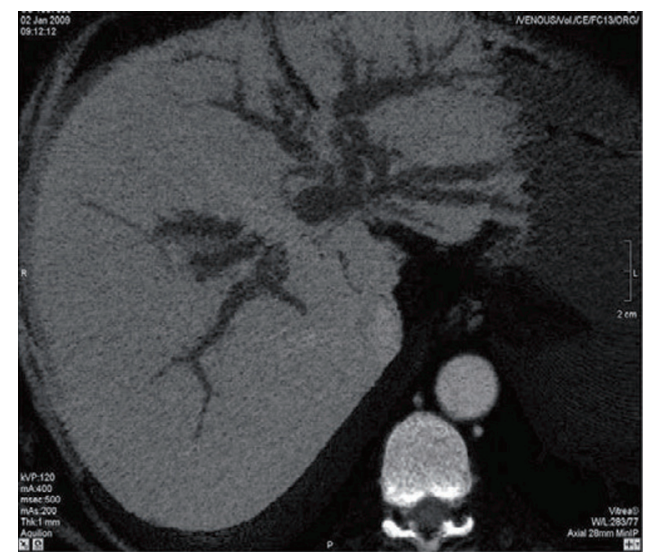

Figure 1 CECT of the abdomen showing dilated biliary radicals in both lobes with non visualisation of the confluence. The left lobe volume was calculated to be $28 \%$. 


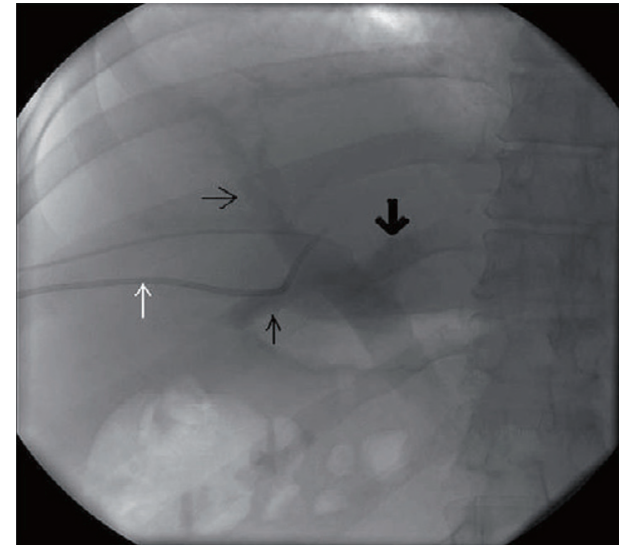

Figure 2 Pre-embolisation portal venogram. The main portal vein as well as the right (thin black arrow) and left lobe (thick black arrow) branches are seen. The previously placed percutaneous transhepatic biliary drainage catheter can also be seen (white arrow).

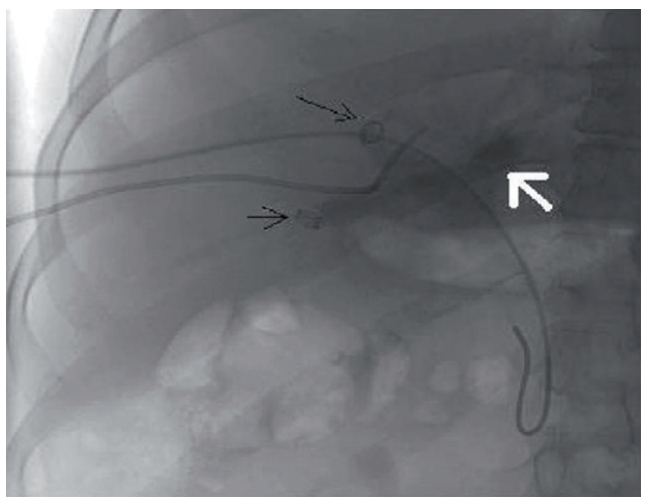

Figure 3 Post-embolisation of the right branch of the portal vein using polyvinyl alcohol particles and embolisation coils. There is no flow of contrast seen distal to the coils (black arrows). The left branch of the portal vein is seen normally (white arrow).

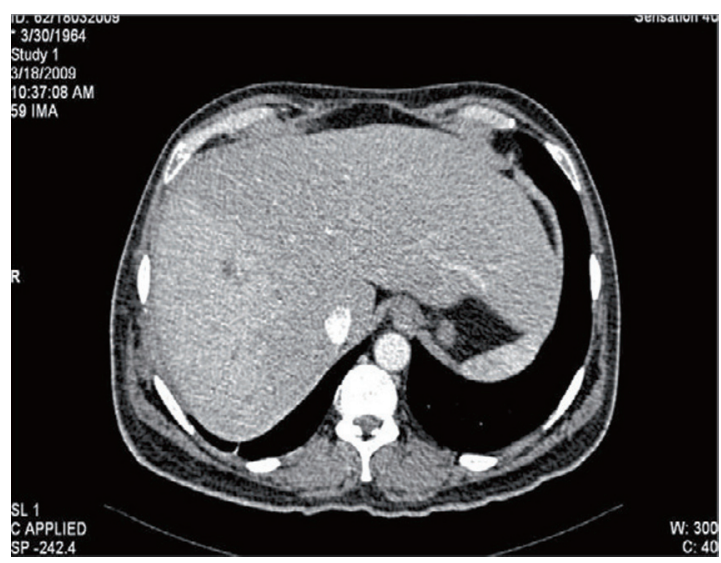

Figure 4 CECT five weeks after portal vein embolisation shows selective hypertrophy of the left lobe which now measures $40 \%$ of the estimated liver volume.

size" liver remnant. The likely cause for this was the fact that the patient had a significantly fatty liver. The patient was managed conservatively and recovered fully on follow-up. The histopathology examination of the resected specimen revealed the lesion to be fibroinflammatory (malignant masquerade) without any evidence of malignancy.

\section{DISCUSSION}

Major hepatectomy carries a significant risk of mortality. In patients with metastases in an otherwise normal functioning liver, mortality after major hepatectomy ranges from $0.5 \%$ to $4 \%$, but in patients with chronic liver disease mortality may be upto $12 \% .^{2,3}$ The main cause of mortality as well as morbidity after major hepatic resection is liver insufficiency often due to an inadequate remnant liver volume. ${ }^{4,5}$ Portal vein embolisation depends on the extraordinary property of the liver to regenerate itself. Early in the 20th century it was noted in animal experiments that if a portal vein branch was occluded, it led to atrophy of the segments supplied by the ligated branches and hypertrophy of the remaining liver. ${ }^{6}$ Portal vein embolisation (PVE) was first described by Kinoshita for hepatocellular carcinoma and later by Makuuchi for hepatic resection of hilar cholangiocarcinomas.? It is now believed that the body can tolerate a residual liver volume of a minimum of $25 \%$, provided that the remnant liver tissue is not affected by diffuse disorders e.g. fatty liver or cirrhosis. ${ }^{8,9}$ Following a major resection, the liver is able to regenerate to sufficient size even if only 2 or 3 adjacent segments remain. This regeneration capacity of the liver depends on two important factors - the capacity of hepatocytes to dedifferentiate and multiply and the response to various growth factors including hepatocyte growth factor (HGF). Incidentally the relatively low amounts of insulin (a growth factor) in diabetics may contribute to slower regeneration rates. ${ }^{10}$

Interruption of portal flow to selected segments may be done surgically (portal vein ligation) or endovascularly (portal vein embolisation). Portal vein embolisation has been reported to result in a significantly more efficient increase in liver volume and a shorter hospital stay. ${ }^{11}$ Two techniques are used for portal vein embolisation: percutaneous transhepatic portal embolisation (PTPE) and transileocolic portal embolisation (TIPE). The increase in remnant liver volume is reported to be greater in PTPE than TIPE group. ${ }^{12}$ The trans hepatic route may be through the ipsilateral approach (through the lobe that is to be embolised) or the contralateral side (through the lobe targeted to hypertrophy). The ipsilateral route may be difficult due to the tumour mass but it avoids risk of injury to the rest of the liver that will form the future liver remnants.

Various materials have been used for embolisation of the portal veins. Use of gelfoam and spherical polyvinyl alcohol (PVA) particles has given suboptimal results. Direct injection of absolute alcohol into the portal vein has been described but is difficult to control and is associated with significant morbidity. Amongst the recommended embolisation agents are a mixture of $n$-butyl-cyanoacrylate (NBCA) and iodized oil (lipidol) or a mixture of fibrin glue with iodised oil. One of the most successful embolisation techniques involves non spherical PVA particles and coils. Smaller size PVA $(300-500 \mu)$ are used to embolise distal branches. This is followed by larger $700-1000 \mu$ 
particles to block more proximal branches. Coils are used at the end of the procedure to allow for complete occlusion of the proximal trunk. It is advisable to avoid all too proximal occlusions and rather leave $1 \mathrm{~cm}$ unembolised segment of the right portal branch in order to facilitate surgical ligation at the time of liver resection.

Patient selection is a very important step to ensure success of the procedure. This decision should be taken in a tumour board meeting including surgeons, hepatologists, oncologists and interventional radiologists. The only contraindication to the liver resection must be insufficient volume of the anticipated future liver remnant. If the FLR is free of disease, portal vein embolisation is offered in case the remnant liver is smaller than $25-40 \%$. In cases of obstructive jaundice, cholestasis reduces the regeneration rate of liver and the biliary obstruction should be relieved prior to the procedure. In such cases FLR of approx $40 \%$ must be targeted. If the FLR to TELV (total estimated liver volume) ratio is less than these values, the patient may be considered for portal vein embolisation. There are almost no absolute contraindications for the procedure except conditions that preclude the subsequent definitive surgery itself. Relative contraindications for PVE include an uncorrectable coagulopathy, tumour invasion of the portal vein, tumour precluding safe transhepatic access, biliary dilatation (in cases of biliary tree obstruction, drainage is recommended) and portal hypertension. ${ }^{10}$

Because of dual blood supply of the liver, portal vein embolisation does not cause frank necrosis thus avoiding the associated complications. Most patients may complain of mild abdominal discomfort and low grade fever for a few days. These need conservative management. There is no consensus on the antibiotic policy. ${ }^{13}$ The other possible complications include transient haemobilia, bleeding and infection. Complications more specific to PVE include the need for re-embolisation, portal vein thrombosis, and portal hypertension. A complication specific to the transileocolic approach is small bowel obstruction. ${ }^{10}$ The other problems related to portal vein embolisation are inadequate response and progression of the disease process.

In conclusion, portal vein embolisation is a relatively safe and effective procedure to induce hypertrophy of the estimated residual liver tissue in cases undergoing partial hepatectomies. A thorough knowledge of the anatomy of the portal system with adherence to well-defined patient selection criteria is essential for the success of this novel procedure.

\section{REFERENCES}

1. Nordlinger B, Peschaud F, Malafosse R. Resection of liver metastases from colorectal cancer-how can we improve results? Colorectal Dis 2003;5:515-517.

2. Farges 0 , Belghiti J, Kianmanesh R, et al. Portal vein embolization before right hepatectomy: prospective clinical trial. Ann Surg 2003; 237:208-217.

3. Fong Y, Fortner J, Sun RL, Brennan MF, Blumgart LH. Clinical score for predicting recurrence after hepatic resection for metastatic colorectal cancer: analysis of 1001 consecutive cases. Ann Surg 1999;230: 309-318.

4. Abdalla EK, Barnett CC, Doherty D, Curley SA, Vauthey JN. Extended hepatectomy in patients with hepatobiliary malignancies with and without preoperative portal vein embolization. Arch Surg 2002;137: 675-680.

5. Yigitler C, Farges O, Kianmanesh R, Regimbeau JM, Abdalla EK, Belghiti J. The small remnant liver after major liver resection: how common and how relevant? Liver Transpl 2003;9:S18-S25.

6. Rous P, Larimore LD. Relation of the portal blood flow to liver maintenance: a demonstration of liver atrophy conditional on compensation. J Exp Med 1920;31:609-632.

7. Alan W, Alan H, Reed I, Howard RJ, et al. Pre-operative portal vein embolization for extended hepatectomy. Ann Surg 2003;237:686-693.

8. Makuuchi M, Thai BL, Takayasu K, et al. Preoperative portal vein embolization to increase safety of major hepatectomy for hilar bile duct carcinoma: a preliminary report. Surgery 1990;107:521-527.

9. De Baere T, Roche A, Elias D, Lasser P, Lagrange C, Bousson V. Pre-operative portal vein embolization for extension of hepatectomy indications. Hepatology 1996;24:1386-1391.

10. Madoff DC, Hicks ME, Vauthey JN, et al. Transhepatic portal vein embolization: anatomy, indications, and technical considerations. RadioGraphics 2002;22:1063-1076.

11. Dieter C, Broering D, Hillert C, Krupski G, et al. Portal vein embolization vs. portal vein ligation for induction of hypertrophy of the future liver remnant. J Gastroint Surg 2002;6:905-913.

12. Abulkhir A, Limongelli P, Healey AJ, et al. Preoperative portal vein embolization for major liver resection: a meta-analysis. Ann Surg 2008;247:49-57.

13. Madoff DC, Abdalla EK, Vauthey JN. Portal vein embolization in preparation for major hepatic resection: evolution of a new standard of care. J Vasc Interv Radiol 2005;16:779-790. 